Si gnal I i ng pat hway i nvol vi ng GULP, MAPK and Rac1 for SR-Bl - i nduced phagocyt osi s of apopt ot i c cell s.

\begin{tabular}{|l|l|}
\hline 著者 & $\begin{array}{l}\text { Osada Yoi chi, Sunat ani Toshi ro, K m I n- San, } \\
\text { Nakani shi Yoshi nobu, Shi rat suchi Aki ko }\end{array}$ \\
\hline $\begin{array}{l}\text { j our nal or } \\
\text { publ i cat } \mathrm{i} \text { on } \mathrm{ti} \text { t l e }\end{array}$ & Journal of bi ochemi st ry \\
\hline vol une & 145 \\
\hline number & 3 \\
\hline page $\mathrm{r}$ ange & $387-394$ \\
\hline year & $2009-03-01$ \\
\hline URL & $\mathrm{ht} \mathrm{t} \mathrm{p:} \mathrm{//hdl} \mathrm{.} \mathrm{handl} \mathrm{e.} \mathrm{net} \mathrm{/2297/18196}$ \\
\hline
\end{tabular}




\title{
Signalling Pathway Involving GULP, MAPK and Rac1 for SR-BI-Induced Phagocytosis of Apoptotic Cells
}

\author{
Yoichi Osada ${ }^{1}$, Toshiro Sunatani ${ }^{1}$, In-San Kim ${ }^{2}$, Yoshinobu Nakanishi ${ }^{1}$ and \\ Akiko Shiratsuchi ${ }^{1, *}$ \\ ${ }^{1}$ Graduate School of Medical Science, Kanazawa University, Kanazawa, Ishikawa 920-1192, Japan; and \\ ${ }^{2}$ Cell and Matrix Research Institute, School of Medicine, Kyungpook National University, Daegu 700-422, \\ Republic of Korea
}

Received September 14, 2008; accepted December 13, 2008; published online January 3, 2009

\begin{abstract}
Class B scavenger receptor type I (SR-BI) is a phosphatidylserine (PS)-recognizing receptor of testicular Sertoli cells responsible for the phagocytosis of spermatogenic cells undergoing apoptosis. Here, we determined signal mediators that compose a signalling pathway for SR-BI-induced phagocytosis. Results of a yeast twohybrid analysis and a cell-free binding assay indicated that SR-BI binds to engulfment adapter protein (GULP) using the C-terminal intracellular domain. A co-immunoprecipitation analysis showed the existence of a complex of GULP and SR-BI in cells prior to the activation of SR-BI by PS. A reduction of GULP expression in phagocytes decreased the SR-BI-mediated phagocytosis of apoptotic cells. Administration to phagocytes of PS-containing liposomes increased the levels of the GTP-bound form of Rac1 and the phosphorylated forms of mitogen-activated protein kinases (MAPK) p38 and extracellular signal-related kinase 1 and 2. Finally, lowering the expression of GULP abrogated MAPK phosphorylation, and the presence of MAPK inhibitors reduced the level of GTP-bound Rac1 in PS-activated phagocytes. These results collectively suggested the following signalling pathway for the SR-BI-induced phagocytosis: (i) PS-recognizing SR-BI activates associated GULP; (ii) activated GULP induces MAPK phosphorylation; (iii) activated MAPK increases GTP-bound Rac1; and (iv) activated Rac1 induces a rearrangement of the actin cytoskeleton.
\end{abstract}

Key words: apoptosis, phagocytosis, phosphatidylserine, scavenger receptor, small G protein.

Abbreviations: CrkII, cell death abnormal-2/chicken tumour virus no. 10 regulator of kinase II; ERK, extracellular signal-related kinase; GST, glutathione $S$-transferase; GULP, engulfment adapter protein; MAPK, mitogen-activated protein kinase(s); PS, phosphatidylserine; siRNA, small interfering RNA; SR-BI, class B scavenger receptor type I.

Cells that have been induced to undergo apoptosis are promptly and selectively eliminated by phagocytosis $(1,2)$, and this mechanism contributes to the architectural and functional establishment of tissues and organs during early development, the renewal of tissues in matured organisms, the regulation of immune responses including inflammation, and the prevention of illness caused by invading pathogenic microbes or own cells that have become harmful (3-5). The phagocytic removal of apoptotic cells also helps physiologic tissue functions to proceed effectively: we previously found that the phagocytosis of male germ cells that have become apoptotic during spermatogenic differentiation is required for the efficient production of sperm (6-8). Apoptotic spermatogenic cells are phagocytosed by a type of testicular somatic cell called the Sertoli cell in a manner mediated by the phagocytosis receptor class B scavenger receptor type I (SR-BI) (7-10), a multiligand receptor also known

*To whom correspondence should be addressed. Tel: +81-76-2344424, Fax: +81-76-264-6223,

E-mail: shira@kenroku.kanazawa-u.ac.jp as a receptor for high-density lipoprotein (11). Sertoli cells recognize apoptotic spermatogenic cells through the binding of SR-BI to phosphatidylserine (PS) exposed on the surface of target cells during apoptosis $(7-9,12,13)$. The engagement of SR-BI with PS most likely activates a signalling pathway leading to the induction of phagocytosis in Sertoli cells. In fact, we found that the phosphorylation of mitogen-activated protein kinases (MAPK) p38 and extracellular signal-related kinase 1 and 2 (ERK1/2) is augmented upon stimulation of SR-BI by PS, and that this event is necessary for a maximal level of phagocytosis (14). However, other signal mediators that compose the signalling pathway activated by SR-BI remain to be identified.

The genetic studies of Horvitz and his colleagues (15) have revealed the existence of two partly overlapping pathways for the induction of phagocytosis in Caenorhabditis elegans phagocytes. One pathway consists of the phagocytosis receptor CED-1, the adaptor protein CED-6 and the Rho family small G protein CED-10, while the other is composed of the adaptor CED-2, the signal mediators CED-5 and CED-12 and CED-10 (15-18); the two pathways seem to converge 
at CED-10 (19). All these CED proteins have counterparts in Drosophila and mammals, suggesting that the two signalling pathways are evolutionally conserved beyond species $(18,20)$. In the present study, we examined which of the two pathways is adopted for the phagocytosis initiated by SR-BI.

\section{MATERIALS AND METHODS}

Materials-The anti-rat SR-BI monoclonal antibody 3D12 and its isotype-matched control 2F2 (mouse IgG1) were generated and characterized as described previously (10). 3D12 specifically recognizes and neutralizes rat SR-BI (no cross-reactivity to mouse SR-BI), while $2 \mathrm{~F} 2$ generated by the same immunization does not bind to SR-BI. Polyclonal anti-rat SR-BI (9) and anti-mouse engulfment adapter protein (GULP) (21) rabbit antisera were generated as described previously. The monoclonal anti-SR-BI antibodies were used to detect rat SR-BI in flow cytometry and immunofluorescence as well as to antagonize the action of rat SR-BI, while the anti-SR-BI antiserum was used to detect rat SR-BI in western blotting. Other antibodies used in this study are as follows: anti-Rac1 (Upstate Biotechnology, Inc., Lake Placid, NY, USA), anti-phosphorylated p38 (Cell Signaling Technology Inc., Beverly, MA, USA), anti-p38 (both the phosphorylated and unphosphorylated forms) (Cell Signaling Technology Inc.), anti-phosphorylated ERK1/2 (Cell Signaling Technology Inc.), anti-ERK1/2 (both the phosphorylated and unphosphorylated forms) (Cell Signaling Technology Inc.), anti-(His)6 (Santa Cruz Biotechnology, Inc., Santa Cruz, CA, USA) and antiglutathione $S$-transferase (GST) (Amersham Biosciences, Uppsala, Sweden). PS and phosphatidylcholine were purchased from Avanti Polar Lipids (Alabaster, AL, USA), and liposomes consisting of phosphatidylcholine and PS at a molar ratio of 7:3 were prepared as described (12). SB203580 and U0126, which respectively inhibit the function of p38 and the phosphorylation of ERK1/2, were purchased from Calbiochem Novabiochem Corp. (San Diego, CA, USA) and Cell Signaling Technology Inc., respectively.

Cells-All experiments involving animals were conducted with the approval of the Committee on Animal Experimentation of Kanazawa University. J774A.1 cells, a mouse macrophage cell line, were cultured in DMEM supplemented with $10 \%(\mathrm{v} / \mathrm{v})$ heat-inactivated fetal bovine serum at $37^{\circ} \mathrm{C}$ with $5 \%(\mathrm{v} / \mathrm{v}) \mathrm{CO}_{2}$ in air. For generating J774A.1 cells ectopically expressing rat SR-BI (J774[SR-BI]), cells were transfected with the plasmid vector pRc/CMV (Invitrogen, Carlsbad, CA, USA) containing cDNA for rat SR-BI (13) using Gene PORTER2 (Genlantis, San Diego, CA, USA). Colonies that had grown in the presence of G418 (Promega, Madison, WI, USA) were analysed for the cell surface expression of rat SR-BI using 3D12 by either flow cytometry or immunofluorescence. Three colonies independently isolated showed almost the same levels of rat SR-BI expression (data not shown), and cells from one colony were used throughout this study. HEK293 cells, a human kidneyderived cell line, were maintained in DMEM supplemented with $10 \%$ heat-inactivated fetal bovine serum at $37^{\circ} \mathrm{C}$ with $5 \% \mathrm{CO}_{2}$ in air. Thymocytes prepared from ddY mice (females, 6- to 10 -weeks old) were induced to undergo apoptosis by incubation with dexamethasone $\left(10^{-5} \mathrm{M}\right)$ in RPMI1640 medium supplemented with $10 \%$ fetal bovine serum, and those cells were efficiently phagocytosed in a PS-dependent manner, as described previously (22).

Assay for Interaction of GULP and SR-BI-A yeast two-hybrid analysis was done using a commercial kit (Clonetech, Mountain View, CA, USA). In brief, the plasmid vector pACT2 containing cDNA coding for amino acid residues 1-8 or amino acid residues 464-509, with the N-terminus numbered as 1 , of rat SR-BI, which respectively corresponded to the $\mathrm{N}$-terminal and C-terminal intracellular domains, was introduced into the Saccharomyces cerevisiae strain PJ69-4a together with the plasmid vector pGBKT7 containing cDNA for cell death abnormal-2/chicken tumour virus no. 10 regulator of kinase II (CrkII) or GULP (a gift from K. Ravichandran, University of Virginia, USA). The occurrence of binding between protein products of each plasmid DNA was examined according to the manufacturer's instructions. For a binding assay using recombinant proteins, the intracellular domains of SR-BI (same as those used in the yeast two-hybrid analysis) tagged with (His)6 were expressed in and purified from Escherichia coli, and CrkII and GULP were similarly prepared from $E$. coli as proteins fused to GST. GSTCrkII, GST-GULP and GST alone $(10 \mu \mathrm{g})$ immobilized on glutathione-Sepharose 4B (Amersham Biosciences) were incubated with the His-tagged SR-BI proteins $(10 \mu \mathrm{g})$ in buffer composed of $25 \mathrm{mM}$ Tris- $\mathrm{HCl}(\mathrm{pH} 7.5), 0.1 \mathrm{M}$ $\mathrm{NaCl}, 2 \mathrm{mM}$ EDTA and $0.5 \%$ (v/v) Triton X-100 for $4 \mathrm{~h}$ at $4^{\circ} \mathrm{C}$. The Sepharose was washed four times with the same buffer, pelleted and incubated with SDS-PAGE buffer for $5 \mathrm{~min}$ at $100^{\circ} \mathrm{C}$ followed by centrifugation. The supernatants were analysed by western blotting with anti-(His) 6 and anti-GST antibodies. For a co-immunoprecipitation analysis, HEK293 cells were transfected with pRc/CMV expressing rat SR-BI and p3XFLAG-CMV-10 (Sigma-Aldrich, St Louis, MO, USA) expressing FLAG-tagged human GULP by the calcium/ phosphate method. They were subsequently incubated in the absence and presence of PS-containing liposomes $(0.05 \mathrm{mM})$ and lysed with a buffer consisting of $10 \mathrm{mM}$ Tris-HCl (pH7.5), $150 \mathrm{mM} \mathrm{NaCl}, 1 \mathrm{mM}$ EDTA, $1 \%$ (v/v) NP-40, 0.1\% (w/v) sodium deoxycholate, $0.1 \%(\mathrm{w} / \mathrm{v})$ SDS, $5 \mu \mathrm{g} / \mathrm{ml}$ leupeptin and $5 \mu \mathrm{g} / \mathrm{ml}$ aprotinin. The cell lysates were mixed with protein G-Sepharose (Amersham Biosciences), and materials bound by the Sepharose were analysed by western blotting with anti-FLAG antibody (Sigma-Aldrich).

Assays for Phagocytosis-Phagocytosis reactions were carried out essentially as described (22). In brief, J774[SR-BI] cells and apoptotic thymocytes as phagocytes and targets, respectively, were mixed at a ratio of 1:10 and incubated for $30 \mathrm{~min}$ at $37^{\circ} \mathrm{C}$. The cultures were then washed with phosphate-buffered saline containing $0.05 \%$ $(\mathrm{w} / \mathrm{v})$ trypsin to remove the thymocytes free or attached to the phagocytes, fixed, stained with haematoxylin and examined for the occurrence of phagocytosis by light-field microscopy. For the determination of a role for Rac1, 
J774[SR-BI] cells were transfected with a plasmid containing cDNA for the dominant negative form of Rac1 (Rac1N17) (23) (a gift from Y. Takuwa, Kanazawa University, Japan) or the empty vector pME18S together with Venus-expressing pCS2 (24) (a gift from A. Miyawaki, RIKEN, Japan) using FuGENE HD transfection reagent (Roche Diagnostics, Indianapolis, IN, USA). Phagocytosis reactions using those J774[SR-BI] cells as phagocytes were carried out as described above, and the washed phagocytes were treated with the DNAbinding fluorochrome Hoechest33342 to visualize the nuclei of engulfed thymocytes followed by examination under a fluorescence-phase contrast microscope. J774[SRBI] cells positive for Venus, a variant of yellow fluorescence protein (24), were considered as those expressing Rac1N17 and examined for the presence of the nuclei of engulfed thymocytes. In all phagocytosis reactions, the level of phagocytosis was exhibited as the phagocytic index: the ratio (in percentage terms) of phagocytes that have accomplished phagocytosis.

Small Interfering RNA-mediated RNA InterferenceSmall interfering RNA (siRNA) for the inhibition of GULP expression was synthesized using a commercial kit (Dicer siRNA Generation kit; Genlantis). The region covering nucleotide positions 348-1019, with the first nucleotide for the translation start codon numbered as 1 , of mouse GULP cDNA placed in front of the T7 promoter was synthesized by PCR, and the GULP sequence was transcribed in vitro and processed by dicer (21). siRNA containing a sequence of green fluorescence protein was similarly generated. J774[SR-BI] cells were transfected with the siRNA for 1-2 days using Gene Silencer reagent (Genlantis) and used in the subsequent experiments.

Determination of the Levels of Phosphorylated MAPK and GTP-bound Rac1-J774[SR-BI] cells or HEK293 cells forcedly expressing rat SR-BI and FLAG-tagged human GULP were incubated in the absence and presence of PS-containing liposome $(0.05 \mathrm{mM})$, and their lysates were analysed by western blotting for the level of phosphorylated MAPK as described previously (14). To determine the level of the GTP-bound form of Rac1, J774[SR-BI] cells after incubation with PS-containing liposomes $(0.05 \mathrm{mM})$ were lysed with $50 \mathrm{mM}$ Tris$\mathrm{HCl}(\mathrm{pH}$ 7.2) buffer containing $0.5 \mathrm{M} \mathrm{NaCl}, 10 \mathrm{mM}$ $\mathrm{MgCl}_{2}, 1 \%$ Triton $\mathrm{X}-100,0.5 \%$ sodium deoxycholate, $0.1 \% \mathrm{SDS}, 5 \mu \mathrm{g} / \mathrm{ml}$ each of leupeptin and aprotinin and $0.1 \mathrm{mM}$ phenylmethylsulfonyl fluoride, and the lysates were centrifuged at $18,000 \mathrm{~g}$ at $4^{\circ} \mathrm{C}$. The supernatants were incubated with glutathione-Sepharose containing p21-activated kinase (25), which preferentially binds to GTP-bound Rac1 (26), for $45 \mathrm{~min}$ at $4^{\circ} \mathrm{C}$. The Sepharose was pelleted, washed with $50 \mathrm{mM}$ Tris-HCl (pH7.2) containing $150 \mathrm{mM} \mathrm{NaCl}, 10 \mathrm{mM} \mathrm{MgCl}_{2}, 1 \%$ Triton $\mathrm{X}-100,5 \mu \mathrm{g} / \mathrm{ml}$ each of leupeptin and aprotinin and $0.1 \mathrm{mM}$ phenylmethylsulfonyl fluoride, and incubated with SDS-PAGE buffer for $5 \mathrm{~min}$ at $100^{\circ} \mathrm{C}$. The proteins released from the Sepharose were analysed by western blotting using anti-Rac1 antibody.

Western Blotting-Proteins separated by SDS-PAGE were transferred to polyvinylidene difluoride membranes and subsequently reacted with primary antibodies and alkaline phosphatase-conjugated secondary antibodies.
The signals were visualized after a chemiluminescence reaction using Immun-Star (Bio-Rad Laboratories, Hercules, CA, USA), and the data were processed in Fluor-S MultiImager (Bio-Rad Laboratories).

Data Processing-Data are representative of at least three independent experiments that yielded similar results. Data from quantitative analyses were expressed as the mean $\pm \mathrm{SD}(n>3)$.

\section{RESULTS}

Binding of GULP to C-terminal Intracellular Region of SR-BI-GULP (mammalian homologue of $C$. elegans CED-6) and CrkII (homologue of CED-2) are adaptor molecules presumably located proximal downstream of membrane-bound receptors in two evolutionally conserved signalling pathways for the induction of phagocytosis of apoptotic cells. We therefore examined if these adaptors bind to the intracellular regions of SR-BI. There are two intracellular domains of rat SR-BI corresponding to amino acid residues $1-8$ and 464-509 with the $\mathrm{N}$-terminus numbered as 1 , and the binding of GULP and CrkII to these two regions was tested in a yeast twohybrid analysis. The results indicated that GULP bound to the $\mathrm{C}$-terminal region, but not to the $\mathrm{N}$-terminal region, of SR-BI (Table 1). We were unable to judge CrkII because an indicator in the two-hybrid analysis became positive in yeast after the expression of CrkII alone (Table 1). On the other hand, ELMO (homologue of CED12) and Rac1 (homologue of CED-10) were negative for the binding to the intracellular regions of SR-BI at least as assessed by the yeast two-hybrid analysis (data not shown). To confirm the results of the yeast two-hybrid analysis, the binding of GULP and CrkII to SR-BI was examined in a cell-free assay using purified recombinant proteins. GULP and CrkII expressed as proteins fused to GST were incubated with the $\mathrm{N}$-terminal and $\mathrm{C}$-terminal intracellular regions of SR-BI tagged with (His)6. The GST-fused proteins were recovered using glutathioneSepharose, and the presence of the (His)6-tagged proteins were examined by western blotting with antibody recognizing the (His)6 tag. We found that the C-terminal region of SR-BI was pulled down on incubation with GST-GULP, while the amount of the N-terminal region precipitated with GST-GULP was at the background level (Fig. 1A). In contrast, GST-CrkII did not seem to

Table 1. Summary of yeast two-hybrid analysis for the binding of SR-BI to GULP and CrkII.

\begin{tabular}{lcccc}
\hline & \multicolumn{4}{c}{ Expression with pACT2 } \\
\cline { 2 - 5 } & SR-BI-N & SR-BI-C & None & largeT \\
\hline Expression & with pGBKT7 & & & \\
CrkII & $+{ }^{\mathrm{b}}$ & + & + & $\mathrm{ND}^{\mathrm{c}}$ \\
GULP & - & ++ & - & $\mathrm{ND}$ \\
none & - & - & - & - \\
p53 & $\mathrm{ND}$ & $\mathrm{ND}$ & - & ++ \\
\hline
\end{tabular}

${ }^{\mathrm{a}}$ The indicated proteins in combination were expressed in yeast: SR-BI-N, N-terminal 9-amino acid region of rat SR-BI; SR-BI-C, C-terminal 46-amino acid region of rat SR-BI; largeT, SV40 large T antigen. ${ }^{\mathrm{b}}$ The level of $\beta$-galactosidase expression in yeast is arbitrarily shown: - , not detected; + , moderate level; ++ , higher level. ${ }^{\mathrm{c}}$ Not done. 
A

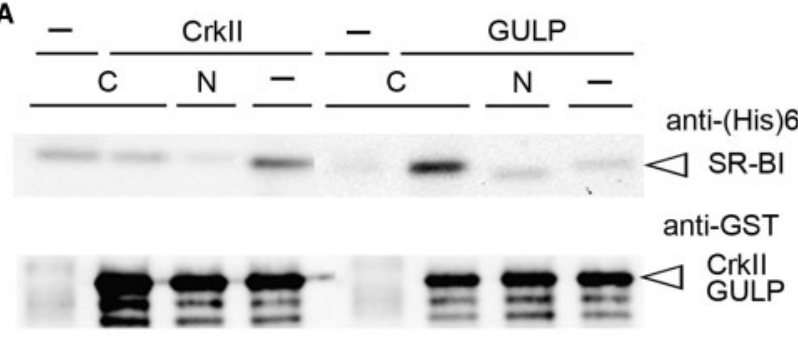

B
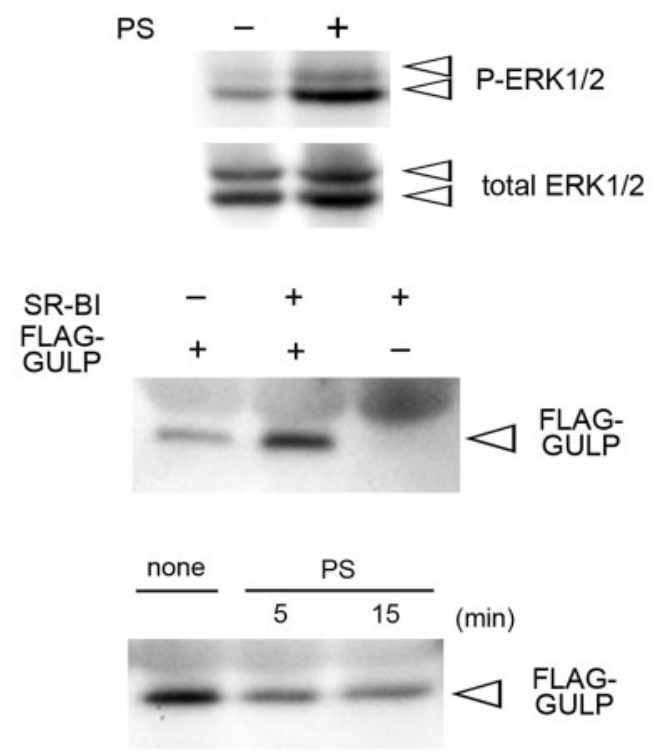

Fig. 1. Binding of GULP, but not CrkII, to C-terminal region of SR-BI. (A) GST-fused CrkII or GULP were incubated with (His)6-tagged SR-BI proteins, pulled down with glutathione-Sepharose, and analysed by western blotting with anti-(His) 6 and anti-GST antibodies. The arrowheads point to the positions of (His)6-tagged SR-BI proteins, and GST-fused CrkII and GULP. C, C-terminal 46-amino acid region of rat SR-BI; N, N-terminal 9-amino acid region of rat SR-BI. (B) HEK293 cells forcedly expressing SR-BI and FLAG-tagged GULP were incubated in the presence or absence of PScontaining liposomes for $10 \mathrm{~min}$, and their whole-cell lysates ( $25 \mu \mathrm{g}$ proteins) were analysed by western blotting for the levels of phosphorylated (P) and both phosphorylated and unphosphorylated (total) ERK1/2 (top panel). HEK293 cells transfected with DNA expressing the indicated proteins were subjected to a co-immunoprecipitation analysis with anti-SR-BI antibody (immunoprecipitation) and anti-FLAG antibody (western blotting) (middle panel). HEK293 cells expressing both SR-BI and FLAG-tagged GULP were incubated with PS-containing liposomes for the indicated periods (PS) or left untreated (none), and their whole-cell lysates were similarly analysed by co-immunoprecipitation (bottom panel).

bind to either the $\mathrm{C}$-terminal or $\mathrm{N}$-terminal intracellular region of SR-BI (Fig. 1A). We next examined the formation of a complex between SR-BI and GULP in HEK293 cells that forcedly expressed these two proteins. The level of phosphorylated ERK1/2 was increased after the incubation with PS-containing liposomes (top panel in Fig. 1B), indicating that SR-BI acts as a PS receptor in these cells. A co-immunoprecipitation analysis with whole-cell lysates confirmed the presence of the complex (middle panel in Fig. 1B), and its level did not seem to change before and after the treatment of cells with
PS-containing liposomes (bottom panel in Fig. 1B). These results collectively showed that GULP binds to the C-terminal intracellular region of rat SR-BI, and suggested that GULP not CrkII is an adaptor acting proximal downstream of the phagocytosis receptor SR-BI.

Requirement of GULP for SR-BI-mediated Phagocytosis of Apoptotic Cells-In order to examine the involvement of GULP in the SR-BI-mediated phagocytosis of apoptotic cells, we established an assay for phagocytosis using a mouse macrophage-derived cell line that ectopically expressed rat SR-BI as phagocytes. This cell line, which we named J774[SR-BI], expressed rat SRBI at the cell surface (Fig. 2A) and phagocytosed apoptotic thymocytes in a manner dependent on rat SR-BI (Fig. 2B). We then examined the phagocytosis of apoptotic cells by J774[SR-BI] cells in which the expression of GULP was reduced by siRNA. The introduction of siRNA containing a sequence of GULP mRNA brought about a near 50\% decrease in the level of phagocytosis, while siRNA with a control green fluorescence protein sequence had a marginal effect (Fig. 2C). These results indicated that the presence of GULP is necessary for the SR-BI-mediated phagocytosis of apoptotic cells, and suggested that GULP not only binds to SR-BI but also transmits a signal for the induction of phagocytosis.

Involvement of Rac1 in SR-BI-mediated Phagocytosis of Apoptotic Cells-Rac1, a mammalian homologue of C. elegans CED-10 and a member of the Rho family of small G proteins, is responsible for the rearrangement of the actin cytoskeleton that directly leads to engulfment by phagocytes (27-30). We previously showed that the SR-BI-mediated phagocytosis of apoptotic cells required the formation of pseudopods through the reorganization of actin filaments (14). The involvement of Rac1 in the phagocytosis of apoptotic cells by J774 [SR-BI] cells was thus examined. The level of GTP-bound Rac1 increased when J774[SR-BI] cells were incubated with either apoptotic thymocytes or PS-containing liposomes (Fig. 3A), and this was abrogated when antibody antagonizing rat SR-BI was present (Fig. 3B). These results suggested that PS-recognizing SR-BI enhances the exchange of GDP and GTP bound by Rac1 or inhibits the conversion of GTP-bound Rac1 into the GDP-bound form through the GTP-hydrolysing activity of the GTPbound and thus active Rac1 (31). We next examined whether or not Rac1 is required for the SR-BI-mediated phagocytosis of apoptotic cells. To do so, J774[SR-BI] cells were transfected with a plasmid expressing a dominant negative form of Rac1 and used in an assay for phagocytosis. The results showed that the introduction of the dominant negative Rac1 caused a significant reduction of phagocytosis (Fig. 3C). In contrast, the dominant negative RhoA, another member of the Rho family, had no effect on the phagocytosis reaction (data not shown). These results indicated that the action of Rac 1 is needed for the SR-BI-mediated phagocytosis of apoptotic cells.

GULP-regulated Activation of MAPK and MAPKmediated Activation of Rac1-We previously reported that either p38 or ERK1/2 is necessary for the SR-BImediated phagocytosis of apoptotic cells (14). We thus examined the requirement of GULP for the phosphorylation of p38 and ERK in J774[SR-BI] cells upon 

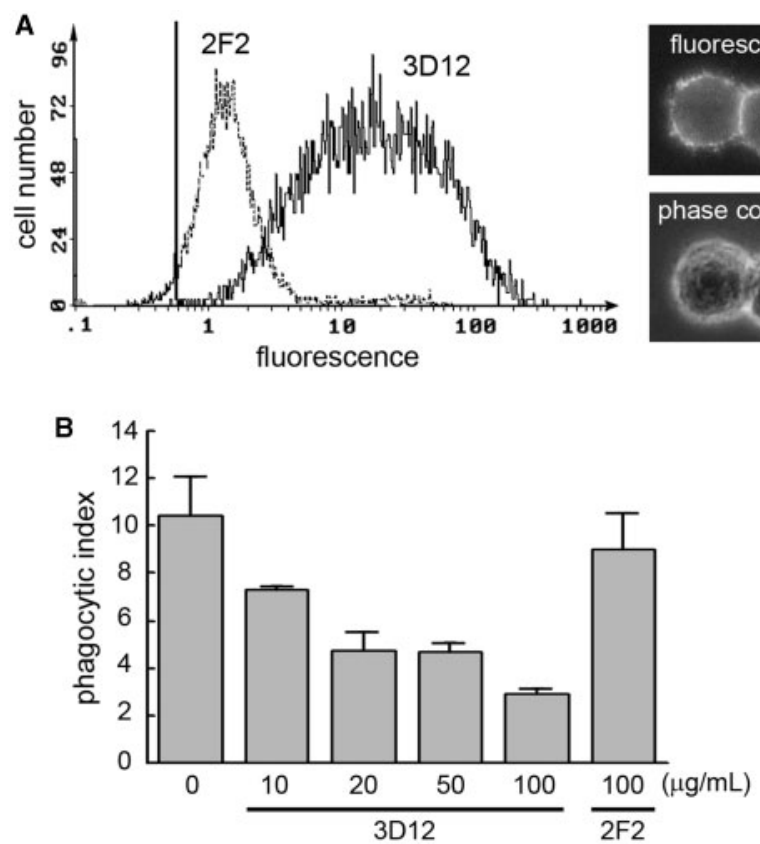

C
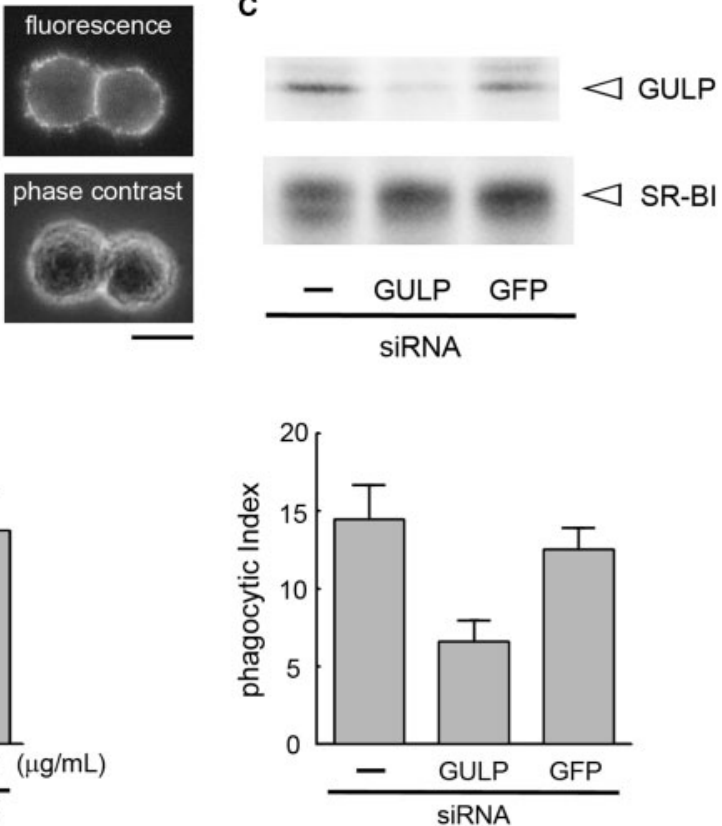

Fig. 2. Requirement of GULP for SR-BI-mediated phagocytosis of apoptotic cells. (A) J774A.1 cells were transfected with a plasmid expressing rat SR-BI, and one of the isolated cell clones was analysed for the surface expression of rat SR-BI by flow cytometry (left) and immunofluorescence (right) using 3D12 and 2F2. The vertical line in the left panel indicates the mean fluorescence intensity with no added antibody. In immunofluorescence, fluorescence and phase-contrast views of the same microscopic filed are shown, and the scale bar indicates $10 \mu \mathrm{m}$.

stimulation by PS-containing liposomes. The incubation of J774[SR-BI] cells with PS-containing liposomes brought about an increase in the level of phosphorylated p38 and ERK, and this effect of the liposomes was significantly mitigated when the level of GULP expression was reduced with siRNA (Fig. 4A). Furthermore, the presence of inhibitors of p38 and ERK abolished the activation of Rac1 in J774[SR-BI] cells treated with PScontaining liposomes (Fig. 4B). These results indicated that the presence of GULP is necessary for the efficient phosphorylation of p38 and ERK, and that the actions of p38 and/or ERK are in turn required for the activation of Rac1.

\section{DISCUSSION}

We showed that SR-BI binds to the CED-6 homologue GULP and functionally interacts with GULP and the CED-10 homologue Rac1. This suggests that SR-BI activates a signalling pathway corresponding to the pathway CED-1-CED-6-CED-10 in C. elegans. We propose the signalling axis SR-BI-GULP-MAPK-Rac1 for the SR-BI-mediated phagocytosis of apoptotic cells: PSbound SR-BI activates associated GULP; activated GULP induces the phosphorylation of p38 and/or ERK; activated MAPK stimulates the production of GTP-bound Rac1; and activated Rac1 induces the rearrangement of actin filaments that subsequently leads to phagocytosis. Although we found that MAPK, p38 and/or ERK1/2, are
(B) Phagocytosis of apoptotic thymocytes by J774[SR-BI] cells was examined in the presence of the indicated antibody. (C) J774[SR-BI] cells were transfected with siRNA containing a sequence of GULP or green fluorescence protein (GFP), or left untreated. Those cells were analysed for the levels of GULP and SR-BI by western blotting (75 $\mu \mathrm{g}$ proteins of whole-cell lysates were used) (top) and for the phagocytic activity with apoptotic thymocytes as targets (bottom).

located downstream of GULP and upstream of Rac1 in the SR-BI-initiated pathway, it is not known at present how these MAPK relate with the other signal mediators in this pathway. An additional signal mediator most likely exists between GULP and MAPK because the adaptor GULP seems unable to phosphorylate MAPK. Similarly, another molecule like a guanine nucleotideexchanging factor or GTPase-activating protein (31) is necessary proximal upstream of Rac1. In line with our findings, Seetharam et al. (32) reported that SR-BI when activated by high-density lipoprotein induces the phosphorylation of MAPK, which eventually leads to the Rac-mediated migration of endothelial cells. Further investigation will be needed to identify the missing components and obtain a complete view of the signalling pathway for the induction of SR-BI-mediated phagocytosis.

PS is the best-characterized marker of phagocytosis that renders many types of cells susceptible to phagocytosis upon the induction of apoptosis $(33,34)$. Besides SRBI, several PS-recognizing phagocytosis receptors have been identified. Kim and colleagues reported that like SR-BI, PS-bound stabilin-2 binds to GULP and transmits a signal for the induction of phagocytosis $(21,35)$. In contrast, another PS receptor BAI1 binds to the mammalian homologue of CED-12 and transmits a signal for phagocytosis via the mammalian homologue of CED- 5 and Rac1 (36). BAI1 thus appears to activate a pathway corresponding to the CED-2/CED-5/CED-12-CED-10 axis 
A

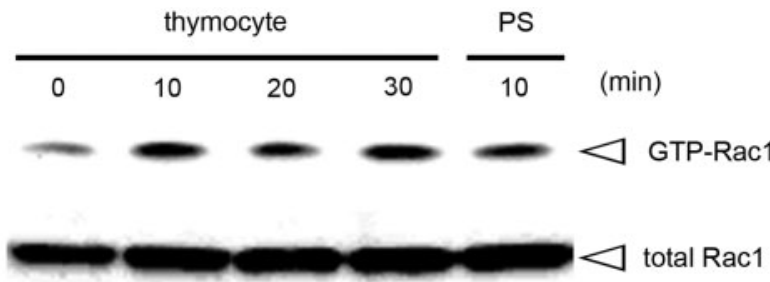

B

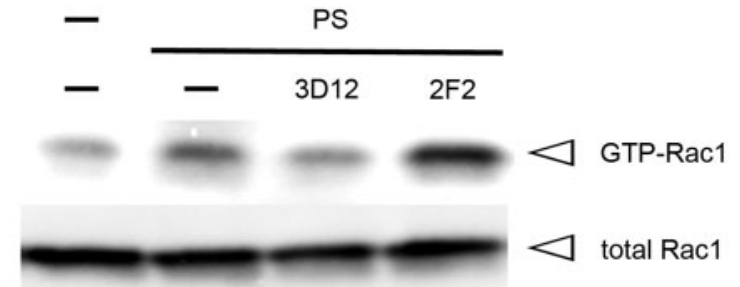

C

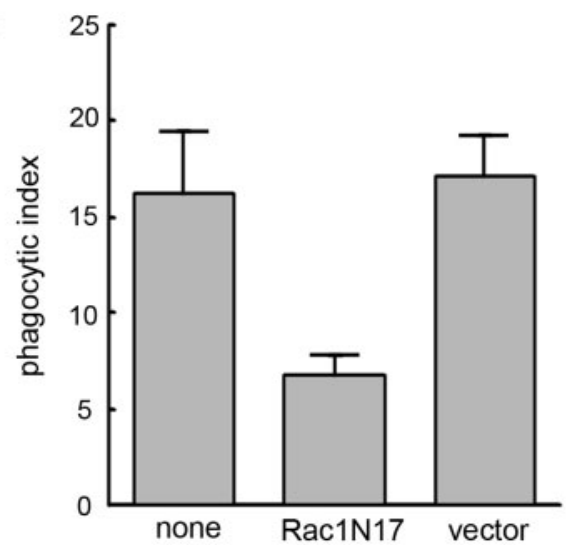

Fig. 3. Requirement of Rac1 for SR-BI-mediated phagocytosis of apoptotic cells. (A) J774[SR-BI] cells were incubated with apoptotic thymocytes (1:10) or PS-containing liposomes (PS) for the indicated periods, and their whole-cell lysates were subjected to an assay for GTP-bound Rac1. The arrowheads point to the positions of GTP-bound Rac1, and both GTP-bound and GDP-bound Rac1 (total Rac1). (B) The same assay shown in A was done in the presence of the indicated antibody $(0.1 \mathrm{mg} / \mathrm{ml})$. (C) J774[SR-BI] cells that had been transfected with a plasmid expressing the dominant-negative form of Rac1 (Rac1N17) or empty vector were subjected to an assay for phagocytosis with apoptotic thymocytes as targets.

in C. elegans. On the other hand, $\alpha \mathrm{v} \beta 5$ integrin (37) and Mer receptor tyrosine kinase (38), which indirectly recognize $\mathrm{PS}$ with the aid of the bridging molecules milk fat globule EGF factor 8 (39) and Gas6 (40), respectively, seem to prefer the pathway CED-2/CED-5/ CED-12-CED-10. These findings suggest that signalling pathways are defined not by the phagocytosis marker or eat-me signal, PS in this case, but by the structural nature of the phagocytosis receptor; that is, which adaptor GULP (CED-6) or CrkII (CED-2) the receptors may bind to. There exist more PS-recognizing receptors, Tim4 (41, 42) and kidney injury molecule-1 (43), as well as other receptors recognizing phagocytosis markers other than PS (44) although the signalling pathways activated by these receptors remain to be characterized. It is important to identify proteins responsible for
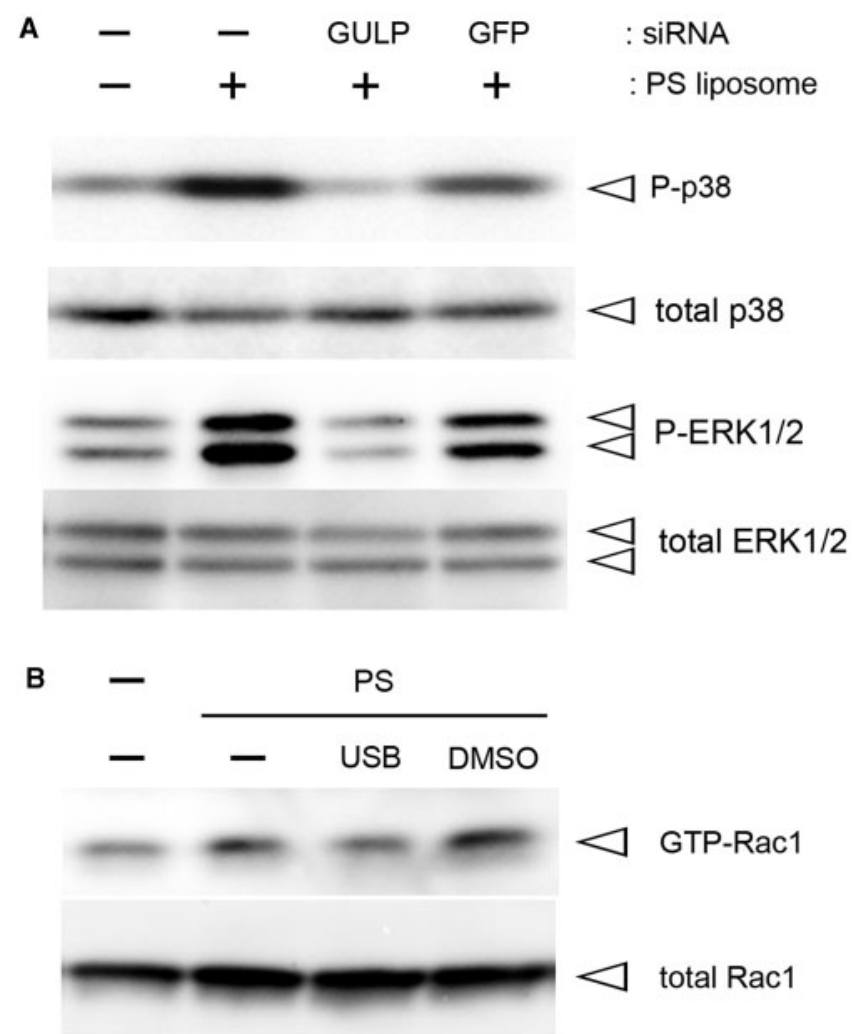

Fig. 4. GULP-dependent phosphorylation of p38 and ERK, and MAPK-dependent activation of Rac1. (A) J774A.1 [SR-BI] cells that had been transfected with siRNA containing a sequence of GULP or green fluorescence protein (GFP), or left untreated were incubated with PS-containing liposomes for $15 \mathrm{~min}$ (for the analysis of p38) or $10 \mathrm{~min}$ (for the analysis of ERK), and their whole-cell lysates (50 $\mathrm{g}$ proteins for p38 and $25 \mu \mathrm{g}$ proteins for ERK) were analysed by western blotting for the amounts of phosphorylated (P), and both phosphorylated and unphosphorylated (total) MAPK. (B) J774A.1[SR-BI] cells were incubated with PS-containing liposomes (PS) in the presence of SB203580 $(2 \mu \mathrm{M})$ and U0126 $(10 \mu \mathrm{M})$ (USB) or solvent alone (DMSO) for $60 \mathrm{~min}$, and their whole-cell lysates were subjected to an assay for GTP-bound Rac1. The arrowheads point to the positions of GTP-bound Rac1, and both GTPbound and GDP-bound Rac1 (total Rac1).

transmitting a phagocytosis-inducing signal initiated by those receptors and determine which of the two evolutionally conserved pathways the corresponding signalling pathways belong to. Such efforts will be required to gain an integrated view of the mechanism by which phagocytes start to engulf apoptotic cells.

\section{ACKNOWLEDGEMENTS}

We thank Y. Takuwa, A. Miyawaki and K. Ravichandran for the materials, and K. Yoshioka and M. Ichiki of Kanazawa University for help in a yeast two-hybrid analysis and an assay for Rac1 activation, respectively.

\section{FUNDING}

Scientific Research from Ministry of Education, Culture, Sports, Science and Technology-Japan (18057009 to A.S.) 
and for Scientific Research from Japan Society for the Promotion of Science (18570123 and 20570127 to A.S.) in part.

\section{CONFLICT OF INTEREST}

None declared.

\section{REFERENCES}

1. Wyllie, A.H., Kerr, J.F.R., and Currie, A.R. (1980) Cell death: the significance of apoptosis. Int. Rev. Cytol. 68, 251-306

2. Ellis, R.E., Yuan, J., and Horvitz, H.R. (1991) Mechanisms and functions of cell death. Annu. Rev. Cell Biol. 7, 663-698

3. Savill, J. and Fadok, V. (2000) Corpse clearance defines the meaning of cell death. Nature 407, 784-788

4. Liao, D.J. (2005) The scavenger cell hypothesis of apoptosis: apoptosis redefined as a process by which a cell in living tissue is destroyed by phagocytosis. Med. Hypotheses 65, 23-28

5. Nakanishi, Y., Henson, P.M., and Shiratsuchi, A. (2006) Pattern recognition in phagocytic clearance of altered self in Target Pattern Recognition in Innate Immunity (Kishore, U., ed.) Chapter 11, Landes Bioscience, Austin

6. Maeda, Y., Shiratsuchi, A., Namiki, M., and Nakanishi, Y. (2002) Inhibition of sperm production in mice by annexin V microinjected into seminiferous tubules: possible etiology of phagocytic clearance of apoptotic spermatogenic cells and male infertility. Cell Death Differ. 9, 742-749

7. Nakanishi, Y. and Shiratsuchi, A. (2004) Phagocytic removal of apoptotic spermatogenic cells by Sertoli cells: mechanisms and consequences. Biol. Pharm. Bull. 27, 13-16

8. Nakagawa, A., Shiratsuchi, A., Tsuda, K., and Nakanishi, Y. (2005) In vivo analysis of phagocytosis of apoptotic cells by testicular Sertoli cells. Mol. Reprod. Dev. 71, 166-177

9. Shiratsuchi, A., Kawasaki, Y., Ikemoto, M., Arai, H., and Nakanishi, Y. (1999) Role of class B scavenger receptor type I in phagocytosis of apoptotic rat spermatogenic cells by Sertoli cells. J. Biol. Chem. 274, 5901-5908

10. Nakagawa, A., Nagaosa, K., Hirose, T., Tsuda, K., Hasegawa, K., Shiratsuchi, A., and Nakanishi, Y. (2004) Expression and function of class B scavenger receptor type I on both apical and basolateral sides of the plasma membrane of polarized testicular Sertoli cells of the rat. Develop. Growth Differ. 46, 283-298

11. Rigotti, A., Miettinen, H.E., and Krieger, M. (2003) The role of the high-density lipoprotein receptor SR-BI in the lipid metabolism and endocrine and other tissues. Endocrine Rev. 24, 357-387

12. Shiratsuchi, A., Umeda, M., Ohba, Y., and Nakanishi, Y. (1997) Recognition of phosphatidylserine on the surface of apoptotic spermatogenic cells and subsequent phagocytosis by Sertoli cells of the rat. J. Biol. Chem. 272, $2354-2358$

13. Kawasaki, Y., Nakagawa, A., Nagaosa, K., Shiratsuchi, A., and Nakanishi, Y. (2002) Phosphatidylserine binding of class B scavenger receptor type I, a phagocytosis receptor of testicular Sertoli cells. J. Biol. Chem. 277, 27559-27566

14. Osada, Y., Shiratsuchi, A., and Nakanishi, Y. (2006) Involvement of mitogen-activated protein kinases in class B scavenger receptor type I-induced phagocytosis of apoptotic cells. Exp. Cell Res. 312, 1820-1830

15. Reddien, P.W. and Horvitz, H.R. (2004) The engulfment process of programmed cell death in Caenorhabditis elegans. Annu. Rev. Cell Dev. Biol. 20, 193-221

16. Kinchen, J.M. and Hengartner, M.O. (2004) Tales of cannibalism, suicide, and murder: programmed cell death in C. elegans. Curr. Top. Dev. Biol. 62, 1-45
17. Mangahas, P.M. and Zhou, Z. (2005) Clearance of apoptotic cells in Caenorhabditis elegans. Semin. Cell Dev. Biol. 16, 295-306

18. Lettre, G. and Hengartner, M.O. (2006) Developmental apoptosis in C. elegans: a complex CEDnario. Nat. Rev. Mol. Cell Biol. 7, 97-108

19. Kinchen, J.M., Cabello, J., Klingele, D., Wong, K., Feichtinger, R., Schnabel, H., Schnabel, R., and Hengartner, M.O. (2005) Two pathways converge at CED10 to mediate actin rearrangement and corpse removal in C. elegans. Nature 434, 93-99

20. Kinchen, J.M. and Ravichandran, K.S. (2007) Journey to the grave: signaling events regulating removal of apoptotic cells. J. Cell Sci. 120, 2143-2149

21. Park, S.Y., Kang, K.B., Thapa, N., Kim, S.Y., Lee, S.J., and Kim, I.S. (2008) Requirement of adaptor protein GULP during stabilin-2-mediated cell corpse engulfment. J. Biol. Chem. 283, 10593-10600

22. Shiratsuchi, A., Watanabe, I., Takeuchi, O., Akira, S., and Nakanishi, Y. (2004) Inhibitory effect of Toll like receptor 4 on fusion between phagosomes and endosomes/lysosomes in macrophages. J. Immunol. 172, 2039-2047

23. Mitsui, H., Takuwa, N., Kurokawa, K., Exton, J.H., and Takuwa, Y. (1997) Dependence of activated G $\alpha 12$-induced $\mathrm{G}_{1}$ to $\mathrm{S}$ phase cell cycle progression on both Ras/mitogenactivated protein kinase and Ras/Rac1/Jun N-terminal kinase cascades in NIH3T3 fibroblasts. J. Biol. Chem. 272, 4904-4910

24. Nagai, T., Ibata, K., Park, E.S., Kubota, M., Mikoshiba, K., and Miyawaki, A. (2002) A variant of yellow fluorescent protein with fast and efficient maturation for cell-biological applications. Nat. Biotechnol. 20, 87-90

25. Okamoto, H., Takuwa, N., Yokomizo, T., Sugimoto, N., Sakurada, S., Shigematsu, H., and Takuwa, Y. (2000) Inhibitory regulation of Rac activation, membrane ruffling, and cell migration by the $G$ protein-coupled sphingosine1-phosphate receptor EDG5 but not EDG1 or EDG3. Mol. Cell. Biol. 20, 9247-9261

26. Benard, V., Bohl, B.P., and Bokoch, G.M. (1999) Characterization of rac and cde42 activation in chemoattractant-stimulated human neutrophils using a novel assay for active GTPases. J. Biol. Chem. 274, 13198-13204

27. Small, J.V., Stradal, T., Vignal, E., and Rottner, K. (2002) The lamellipodium: where motility begins. Trends Cell Biol. 12, $112-120$

28. Ellis, S. and Mellor, H. (2000) Regulation of endocytic traffic by Rho family GTPases. Trends Cell Biol. 10, 85-88

29. Bokoch, G.M. (2005) Regulation of innate immunity by Rho GTPases. Trends Cell Biol. 15, 163-171

30. Côté, J.F. and Vuori, K. (2007) GEF what? Dock180 and related proteins help Rac to polarize cells in new ways. Trends Cell Biol. 17, 383-393

31. Bos, J.L., Rehmann, H., and Wittinghofer, A. (2007) GEFs and GAPs: critical elements in the control of small G proteins. Cell 129, 865-877

32. Seetharam, D., Mineo, C., Gormley, A.K., Gibson, L.L., Vongpatanasin, W., Chambliss, K.L., Hahner, L.D., Cummings, M.L., Kitchens, R.L., Marcel, Y.L., Rader, D.J., and Shaul, P.W. (2006) High-density lipoprotein promotes endothelial cell migration and reendothelialization via scavenger receptor-B type I. Circ. Res. 98, 63-72

33. Fadok, V.A., Bratton, D.L., Frasch, S.C., Warner, M.L., and Henson, P.M. (1998) The role of phosphatidylserine in recognition of apoptotic cells by phagocytes. Cell Death Differ. 5, 551-562

34. Schlegel, R.A. and Williamson, P. (2001) Phosphatidylserine, a death knell. Cell Death differ. 8, 551-563

35. Park, S.-Y., Jung, M.-Y., Kim, H.-J., Lee, S.-J., Kim, S.-Y., Lee, B.-H., Kwon, T.-H., Park, R.-W., and Kim, I.-S. (2008) Rapid cell corpse clearance by stabilin-2, a membrane phosphatidylserine receptor. Cell Death Differ. 15, 192-201 
36. Park, D., Tosello-Trampont, A.C., Elliott, M.R., Lu, M., Haney, L.B., Ma, Z., Klibanov, A.L., Mandell, J.W., and Ravichandran, K.S. (2007) BAI1 is an engulfment receptor for apoptotic cells upstream of the ELMO/Dock180/Rac module. Nature 450, 430-435

37. Akakura, S., Singh, S., Spataro, M., Akakura, R., Kim, J.I., Albert, M.L., and Birge, R.B. (2004) The opsonin MFG-E8 is a ligand for the $\alpha \mathrm{v} \beta 5$ integrin and triggers DOCK180dependent Rac1 activation for the phagocytosis of apoptotic cells. Exp. Cell Res. 292, 403-416

38. Tibrewal, N., Wu, Y., D'mello, V., Akakura, R., George, T.C., Varnum, B., and Birge, R.B. (2008) Autophosphorylation docking site Tyr-867 in Mer receptor tyrosine kinase allows for dissociation of multiple signaling pathways for phagocytosis of apoptotic cells and down-regulation of lipopolysaccharide-inducible NF-. J. Biol. Chem. 283, 3618-3627

39. Hanayama, R., Tanaka, M., Miwa, K., Shinohara, A., Iwamatsu, A., and Nagata, S. (2002) Identification of a factor that links apoptotic cells to phagocytes. Nature 417, 182-187

40. Ishimoto, Y., Ohashi, K., Mizuno, K., and Nakano, T. (2000) Promotion of the uptake of PS liposomes and apoptotic cells by a product of growth arrest-specific gene, gas6. J. Biochem. 127, 411-417

41. Miyanishi, M., Tada, K., Koike, M., Uchiyama, Y., Kitamura, T., and Nagata, S. (2007) Identification of Tim4 as a phosphatidylserine receptor. Nature 450, 435-439

42. Kobayashi, N., Karisola, P., Peña-Cruz, V., Dorfman, D.M., Jinushi, M., Umetsu, S.E., Butte, M.J., Nagumo, H., Chernova, I., Zhu, B., Sharpe, A.H., Ito, S., Dranoff, G., Kaplan, G.G., Casasnovas, J.M., Umetsu, D.T., DeKruyff, R.H., and Freeman, G.J. (2007) TIM-1 and TIM-4 glycoproteins bind phosphatidylserine and mediate uptake of apoptotic cells. Immunity 27, 927-940

43. Ichimura, T., Asseldonk, E.J., Humphreys, B.D., Gunaratnam, L., Duffield, J.S., and Bonventre, J.V. (2008) Kidney injury molecule-1 is a phosphatidylserine receptor that confers a phagocytic phenotype on epithelial cells. J. Clin. Invest. 118, 1657-1668

44. Lauber, K., Blumenthal, S.G., Waibel, M., and Wesselborg, S. (2004) Clearance of apoptotic cells: getting rid of the corpses. Cell 14, 277-287 